\title{
Stress and obesity connect at NPY
}

DOI:

10.1038/nrd2408
Stress is known to be linked to body weight, but the precise nature and molecular mechanisms of this connection remain poorly defined. Some people lose weight and other people gain weight in response to stress. Some centrally acting mechanisms such as the influence of stress and obesity on the hypothalamic control of food intake and metabolism have been described. Reporting in Nature Medicine, Kuo and colleagues now show that the stress-triggered release of neuropeptide Y (NPY) can also directly stimulate fat angiogenesis in the periphery, as well as the proliferation and differentiation of new fat cells. These effects are mediated by the neuropeptide Y2 receptor (NPY2R), which might therefore be an attractive target for anti-obesity drugs.

NPY is a potent orexigenic neurotransmitter derived from the brain and sympathetic nerves. Its release and actions can be modulated by stress hormones - in mice, exposure to physical (cold) or emotional (aggressor alpha-mouse) stress elevates plasma levels of NPY. The authors showed that this stress-induced activation of the NPY system, combined with a high fat and sugar diet (HFS), results in increased abdominal fat deposits in mice and upregulation of the genes for NPY, NPY2R and an enzyme, DPP4 (dipeptidyl peptidase 4), which forms an NPY-Y2R agonist in the white adipose tissue (WAT). In vitro experiments demonstrated that NPY exposure can directly stimulate the proliferation of adipocytes, upregulate $N p y$ and Npy2r, and mimick the effects of insulin, such as increasing the secretion of leptin and resistin, and lipid filling of new adipocytes.

In vivo, genetically obese mice were found to have higher NPY plasma levels and increased NPY and NPY2R expression in subcutaneous abdominal fat. Slow-release pellets of NPY inserted into the fat increased WAT in both lean and obese mice, and human-fat xenografts showed increased survival and vascularization in the presence of an NPY pellet. By contrast, intra-fat injection with NPY2R antagonists led to decreased vascularity and increased endothelial and fat cell apoptosis in the abdominal fat pads.

Similar to the effects of NPY on mouse and human fat, cold or aggression stress in HFS-fed mice resulted in increased vascularity of abdominal WAT, the proliferation and growth of new adipocytes, and the development of metabolic-like syndrome. Treatment with an NPY2R antagonist reduced the abdominal fat deposits by $40 \%$ in these mice within two weeks, and mice with a genetic ablation of NPY2R (Npy2r-1-) were resistant to the stress-induced augmentation of diet-induced obesity (DIO). Npy $2 r^{-1-}$ mice were also resistant to the stress-induced and HFSinduced onset of DIO exacerbations and metabolic-like syndrome.

A conditional $N p y 2 r$-knockout, which allowed genetic ablation in

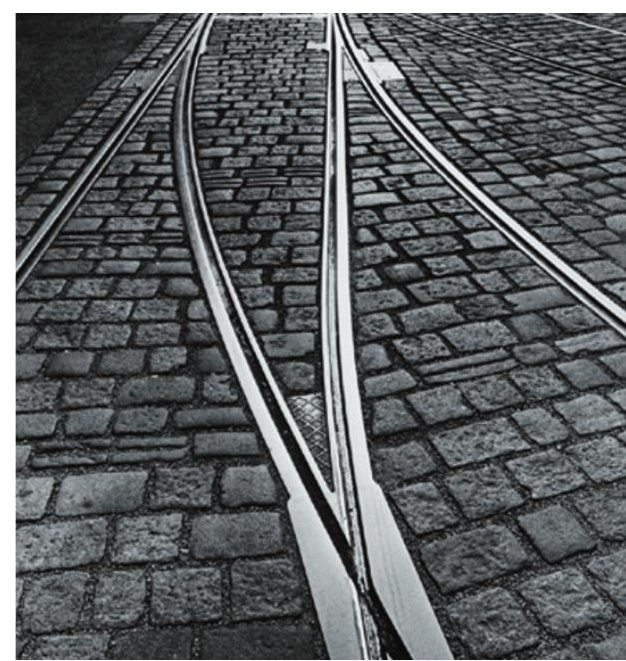

the abdominal WAT, proved that this mechanism is indeed located in the periphery - the local knockdown of NPY2R reduced stress-induced fat by $50 \%$ in two weeks. Interestingly, a silent NPY2R variant has been found in the human population and seems to protect against obesity, and a gain-of function mutant of NPY is associated with obesity, diabetes and accelerated atherosclerosis.

The finding that NPY mediates the weight gain associated with stress and a high-calorie diet suggests novel possibilities for NPY2R-based drugs. NPY2R antagonists might have potential in the treatment and prevention of obesity and metabolic syndrome, as well as 'liporemodelling' such as local non-surgical elimination of fat, whereas NPY/NPY2R agonists might augment the retention of fat grafts.

Alexandra Flemming

ORIGINAL RESEARCH PAPER Kuo, L. E. et al. Neuropeptide $Y$ acts directly in the periphery on fat tissue and mediates stress-induced obesity and metabolic syndrome. Nature Med. 13, 803-811 (2007) 